\title{
Amino acid metabolism, lipid metabolism, and oxidative stress are associated with post-stroke depression: a metabonomics study
}

Man Wang ${ }^{\dagger}$, Xianwei Gui ${ }^{\dagger}$, Lanxiang Wu, Sheng Tian, Hansen Wang, Liang Xie ${ }^{*}$ and Wei Wu ${ }^{*}$

\begin{abstract}
Background: Post-stroke depression (PSD) is a mood disorder characterized by depression and anhedonia caused by stroke. Metabolomics identified metabolites associated with PSD, but previous studies are based on gas chromatography (GC)/mass spectrometry (MS). This study aimed to perform a liquid chromatography (LC)-MS-based metabolomics study of the plasma metabolite profiles between patients with PSD and controls.

Methods: This was a prospective study of patients with stroke enrolled between July and December 2017 at the Second Affiliated Hospital of Nanchang University. Patients were grouped as Hamilton Depression Rating Scale $>7$ (PSD) or $<7$ (controls). Metabonomics profiling of plasma sampled was conducted by LC-MS. By combining multivariable and univariable statistical analyses, significant differential metabolites between the two groups were screened. The threshold for significant differences was VIP $\geq 1$ and $P<0.05 . \log _{2} F C$ is the logarithm of the mean ratio between the two groups.
\end{abstract}

Results: There were no significant difference with respect to age, NIHSS score, and MMSE between the two groups (all $P>0.05$ ). There were six differential metabolites between the PSD and stroke groups, of which three metabolites were increased and three were decreased. Compared with the control group, $\mathrm{p}$-chlorophenylalanine $\left(\log _{2} \mathrm{FC}=1.37\right.$, $P=0.03)$, phenylacetyl glutamine $\left(\log _{2} F C=0.21, P=0.048\right)$, and $D H A\left(\log _{2} F C=0.77, P=0.01\right)$ levels were higher in the PSD group, while betaine (trimethylglycine) ( $\left.\log _{2} F C=-0.79, P=0.04\right)$, palmitic acid ( $\log _{2} F C=-0.51, P=0.001$ ), and $\mathrm{MHPG}-\mathrm{SO}_{4}\left(\mathrm{Log}_{2} \mathrm{FC}=-2.37, P=0.045\right)$ were decreased.

Conclusion: Plasma metabolomics showed that amino acid metabolism (phenylacetyl glutamine, $\mathrm{p}$ chlorophenylalanine, trimethylglycine), lipid metabolism (DHA, palmitic acid, trimethylglycine), and oxidative stress (DHA, palmitic acid, trimethylglycine) were associated with PSD. These results could help to reveal the pathophysiological mechanism of PSD and eventually identify treatment targets.

Keywords: Post-stroke depression, Metabonomics, Liquid chromatography-mass spectrometry, Amino acid metabolism, Lipid metabolism, Oxidative stress

\footnotetext{
* Correspondence: xl580122@163.com; 13807038803@163.com

${ }^{\dagger}$ Man Wang and Xianwei Gui contributed equally to this work. Department of Neurology, the Second Affiliated Hospital of Nanchang University, Nanchang, China
}

C C The Author(s). 2020 Open Access This article is licensed under a Creative Commons Attribution 4.0 International License, which permits use, sharing, adaptation, distribution and reproduction in any medium or format, as long as you give appropriate credit to the original author(s) and the source, provide a link to the Creative Commons licence, and indicate if changes were made. The images or other third party material in this article are included in the article's Creative Commons licence, unless indicated otherwise in a credit line to the material. If material is not included in the article's Creative Commons licence and your intended use is not permitted by statutory regulation or exceeds the permitted use, you will need to obtain permission directly from the copyright holder. To view a copy of this licence, visit http://creativecommons.org/licenses/by/4.0/ The Creative Commons Public Domain Dedication waiver (http://creativecommons.org/publicdomain/zero/1.0/) applies to the data made available in this article, unless otherwise stated in a credit line to the data. 


\section{Background}

Post-stroke depression (PSD) is characterized by depression or severe depressive episode resulting from stroke, with mania or mixed characteristics [1]. The overall incidence of PSD is $31 \%$ at any time within 5 years after stroke. PSD can adversely affect neural functional recovery and significantly increase the disability rate and mortality [2]. Therefore, early identification and treatment of PSD are essential to improve prognosis.

At present, the mechanism of PSD remains unclear. According to the monoamine neurotransmitter imbalance hypothesis, brain damage caused by stroke interferes with ascending projection from the brainstem to the frontal cortex through the thalamus and basal ganglia, which results in reduced bioavailability of serotonin, dopamine, and norepinephrine [3]. Some proinflammatory cytokines may also affect the synthesis and metabolism of amine neurotransmitters: interferon (INF) $\alpha$ can affect the synthesis and reuptake of serotonin [4], and interleukin (IL)-1 $\beta$ and tumor necrosis factor (TNF)- $\alpha$ can activate serotonin transporters to increase serotonin reuptake [5].

Beside neurotransmitters, a number of compounds are associated with PSD. Glutamate is closely related to emotional and mental activities. Compared with stroke patients without PSD, stroke patients with PSD have higher glutamate levels in the frontal lobe [6]. Damage to synaptic plasticity in specific regions of the central nervous system, especially the hippocampus, may be a key factor in the pathophysiology of depression. The brain-derived neurotrophic factor (BDNF) plays an important role in the regulation of synaptic plasticity repair [7]. Decreased serum levels of BDNF are associated with PSD [8]. Elevated serum levels of homocysteine (Hcy) are significantly associated with depression [9], whereas Hcy may affect BDNF expression in non-inflammatory states and endothelial dysfunction [10]. The Nacetylaspartate/creatinine ratio in the hippocampus of PSD patients is lower than in controls, suggesting abnormal metabolism of hippocampal neurons in PSD patients [11].

Metabolomics is defined as the study of metabolic networks by analyzing the dynamic changes in the quality and quantity of metabolites of biosystems stimulated by pathology and physiology [12]. Yang et al. have analyzed the serum metabolomics of schizophrenia patients and identified metabolites associated with schizophrenia [13]. Xiao et al. identified five potential biomarkers of PSD: lactic acid, $\alpha$-hydroxybutyric acid, phenylalanine, formic acid, and arabitol [14]. Zhang et al. found that azelaic acid, glyceric acid, pseudouridine, 5-hydroxycaproic acid, tyrosine, and phenylalanine can be used as biomarkers for the diagnosis of PSD [15]. Ding et al. identified proline, pyroglutamic acid, palmitic acid, oleic acid, linoleic acid, oxalic acid and rhamnose as being associated with PSD [16]. Of note, different methodologies will yield different results simply because they do not look at the same metabolites and those previous studies above are all based on gas chromatography/mass spectrometry (GS/MS).

Liquid chromatography-mass spectrometry (LC-MS) has high sensitivity and requires a simpler sample pretreatment compared with GC/MS [17]. Therefore, the aim of the present study was to perform a LC-MS-based metabolomics study of the plasma metabolite profiles between patients with PSD and controls, in order to identify plasma differential metabolites related to PSD. The results could help identify novel biomarkers of PSD that could ultimately improve prognosis and potentially be treatment targets.

\section{Methods}

\section{Study design and participants}

This was a prospective study of patients with stroke enrolled between July and December 2017 at the inpatient and outpatient clinics of the Department of Neurology of the Second Affiliated Hospital of Nanchang University. This study was approved by the ethics committee of Nanchang University. All patients provided written informed consent to participate in this study. The study was carried out according to the tenets of the Declaration of Helsinki.

The inclusion criteria for the PSD group were: 1) adult patients with confirmed acute stroke (including ischemic and hemorrhagic stroke) within $24 \mathrm{~h}$ after onset by computed tomography (CT) or magnetic resonance imaging (MRI); 2) patients meeting 2014 guidelines for the diagnosis of acute ischemic stroke in China; 3) patients meeting the diagnostic criteria of PSD [1]; 4) Hamilton Depression Rating Scale (HDRS; validated Chinese version [18]) > 7; 5) first-ever onset of stroke; 6) no history of antidepressant drugs; and 7) in the acute stage after stroke (<1 month).

The exclusion criteria were: 1) disturbance of consciousness and/or serious conditions interfering with the examinations; 2) severe dementia, severe aphasia, dysarthria, or deafness; 3) personal or family history of mental disorders; 4) receiving psychiatric treatment; 5) central nervous system infection, head injury, epilepsy, multiple sclerosis, toxic metabolic diseases, Parkinson's disease, intracranial tumor, or hypothyroidism; 6) discharged within 7 days after onset; 7) death within 2 weeks of onset; or 8) severe metabolic diseases (including severe heart, liver, or renal dysfunction, shock, cancer, autoimmune diseases, etc.).

For the control group, the inclusion and exclusion criteria were the same as for the PSD group, except the HDRS being $<7$. 


\section{Plasma sample collection}

Venous blood $(5 \mathrm{ml})$ was drawn from an antecubital vein in EDTA anticoagulated vacuum blood collection tubes (BD Medical, Franklin Lake, NJ, USA) after a 12-h fast. The samples were centrifuged at $1500 \times \mathrm{g}$ for $10 \mathrm{~min}$ at $4{ }^{\circ} \mathrm{C}$. The supernatant plasma was pipetted into sterile Eppendorf tubes, $0.5 \mathrm{ml} /$ tube. The samples were snapfrozen in liquid nitrogen for $30 \mathrm{~s}$ and stored at $-80^{\circ} \mathrm{C}$.

\section{Sample preparation}

About $50 \mathrm{mg}$ of samples and $20 \mu \mathrm{L}$ of internal standard solution were added to $1000 \mu \mathrm{L}$ of extracting solution (methanol:acetonitrile:water $=2: 2: 1$ ) and vortexed for 30 s. Steel beads were added, a $45-\mathrm{Hz}$ grinder (Shanghai Jingxin Science and Technology Co., Ltd., Shanghai, China) was used for $4 \mathrm{~min}$, and ultrasonic extraction (Shenzhen Lei De Bang Electronics Co., Ltd., Shenzhen, China) was performed in ice for $5 \mathrm{~min}$. The extraction were repeated for three times and the samples were left standing $1 \mathrm{~h}$ at $-20^{\circ} \mathrm{C}$. After centrifugation at 13,000 rpm for $15 \mathrm{~min}$ at $4{ }^{\circ} \mathrm{C}, 700 \mu \mathrm{L}$ of the supernatant was taken in an EP tube, dried in a vacuum concentrator, and re-dissolved with $500 \mu \mathrm{L}$ of acetonitrile:water: 1:1. The sample was vortexed for $30 \mathrm{~s}$ and treated in the ultrasonic bath for $10 \mathrm{~min}$. After centrifugation at 13, $000 \mathrm{rpm}$ for $15 \mathrm{~min}$ at $4{ }^{\circ} \mathrm{C}, 60 \mu \mathrm{L}$ of the supernatant was taken out in a $2-\mathrm{mL}$ injection bottle for LC-MS analysis. The quality control (QC) samples were prepared by mixing the experimental sample extracts in equal amounts to analyze the repeatability of the sample under the same treatment method. During analysis, a QC sample was inserted every 6-10 test samples to monitor repeatability.

\section{LC-MS analysis}

The instrument platform for LC-MS analysis included a 1290 Ultra High Performance Liquid Chromatography (Agilent Technologies, Santa Clara, CA, USA) and a Triple TOF 6600 High Resolution Mass Spectrometer (AB SCIEX LLC, Framingham, MA, USA). The column was a UPLC HSS T3 chromatographic column $(1.7 \mu \mathrm{m}$, $2.1 \times 100 \mathrm{~mm}$, Waters, Milford, MA, USA). Mobile phase A was water (containing $25 \mathrm{mM}$ ammonium acetate and $25 \mathrm{mM}$ ammonia water) and mobile phase B was acetonitrile. The gradient elution procedure is shown in Table 1 . The injection volume was $1 \mu \mathrm{L}$.

For MS, data acquisition was under the control of the control software (Analyst TF 1.7, AB SCIEX LLC, Framingham, MA, USA). In each cycle of data acquisition, molecular ions with the highest intensity and greater than 100 were screened for the acquisition of corresponding secondary mass spectral data. Bombardment energy: $30 \mathrm{eV}, 15$ secondary spectrograms per $50 \mathrm{~ms}$. The ESI ion source parameters were set to: atomization
Table 1 Parameters of the mobile phase for LC-MS

\begin{tabular}{llll}
\hline Time $(\mathrm{min})$ & Row $(\mu \mathrm{L} / \mathrm{min})$ & $\mathrm{A} \%$ & $\mathrm{~B} \%$ \\
\hline 0 & 500 & 5 & 95 \\
0.5 & 500 & 5 & 95 \\
7 & 500 & 35 & 65 \\
8 & 500 & 60 & 40 \\
9 & 500 & 60 & 40 \\
9.1 & 500 & 5 & 95 \\
12 & 500 & 5 & 95 \\
\hline
\end{tabular}

pressure (GS1): 60 Psi; auxiliary pressure: 60 Psi; air curtain pressure: $35 \mathrm{Psi}$, temperature: $650^{\circ} \mathrm{C}$, spray voltage: $5000 \mathrm{~V}$ (positive ion mode) or $-4000 \mathrm{~V}$ (negative ion mode) $[19,20]$.

\section{Data analysis}

The plasma metabolite profiles in the PSD and control groups were analyzed. A multidimensional statistical model was established to screen for metabolites with significant differences between the two groups, and to identify biologically significant metabolites related to PSD. The original data after MS were first converted to the mzML format using the ProteoWizard software. XCMS 1.5.0 was used for data processing, such as retention time correction, peak identification, peak extraction, peak integration, and peak alignment. Minfrac was set as 0.5 and cutoff as 0.6. The xcms4dda and xcms4lipid software and a self-built library developed based on XcmMS were used for data filtering. The filtering criterion was that sample number of the metabolite in at least one group of samples was detected to be greater than $50 \%$ $(\operatorname{minfrac}=0.5)$.

\section{Model identification}

Principal component analysis (PCA), partial least squares-discriminant analysis (PLS-DA), and orthogonal partial least-squares discriminant analysis (OPLS-DA) were used. PCA analysis represents an unsupervised multidimensional statistical analysis method that can generally reflect the overall metabolic differences among groups and the degree of variation within groups. PLSDA and OPLS-DA are statistical analysis methods with supervised pattern recognition that can maximize the distinction between groups, thus contributing to the search for differential metabolites (potential biomarkers). Given multivariable statistical analysis would have overfitting, the multivariable statistical analysis model also needed to undergo model verification, permutation test was used to evaluate the accuracy of the (O)PLS models. By combining the multivariable statistical analysis (OPLS-DA variable important in projection (VIP) values) and univariable statistical analyses (Student's t-test), 
significant differential metabolites between the two groups were screened. The threshold for significant difference was: VIP $\geq 1$ and t-test $P<0.05$. The metaboliterelated pathways were searched for using KEGG (http:// www.kegg.jp).

\section{Statistical analysis}

Continuous data are presented as means \pm standard deviation and were analyzed using the Student $t$ test. Categorical data are presented as frequencies and were analyzed using the chi-square test. All statistical analyzes were performed using SPSS 16.0 (IBM, Armonk, NY, USA). Two-sided $P$ values $<0.05$ were considered statistically significant.

\section{Results}

\section{Participants}

Ten stroke patients without PSD and 10 stroke patients with PSD were recruited. There were no significant differences with respect to age, NIHSS score, and MMSE between the two groups (Table 2).

\section{Chromatograms}

Visual examination on the total ion current (TIC) of all samples was conducted. TIC is a chromatogram of the sum of all ionic strengths and the time in the selected mass range. TIC is a reflection of category and abundance of the whole metabolites of samples. The analysis of all samples showed good overlapping and stability of TIC peak retention time and peak area, indicating the reliability of the chromatograms.

\section{Principal component analysis (PCA)}

The PCA model could explain the metabolic differences between the PSD and control groups (Fig. 1).

\section{Partial least squares-discriminant analysis (PLS-DA)} PLS-DA is a multivariable statistical analysis method with supervised pattern recognition. The multidimensional data undergo grouping according to the differential factors that need to be found before compression (the $\mathrm{Y}$ value can be pre-set for target classification and discrimination), so variables that are the most relevant to grouping factors can be found, while reducing

Table 2 Characteristics of the participants

\begin{tabular}{llll}
\hline & Control & PSD & $P$ \\
\hline Age (years) & $57.9 \pm 9.7$ & $61.1 \pm 14.4$ & 0.35 \\
Gender (male/female) & $9 / 1$ & $7 / 3$ & 0.58 \\
HDRS & $2.0 \pm 2.1$ & $15.1 \pm 5.4$ & $<0.001$ \\
NIHSS & $3.6 \pm 4.4$ & $2.8 \pm 3.7$ & 0.39 \\
MMSE & $26.3 \pm 4.4$ & $23.4 \pm 6.5$ & 0.48 \\
\hline
\end{tabular}

HDRS Hamilton Depression Rating Scale; NIHSS National Institutes of Health Stroke Scale; MMSE Mini-Mental State Examination the impact of the other factors. Similar to the PCA analysis, PLS-DA can maximize the distinction between groups, which is helpful to search for differential metabolites. The VIP for a model variable can measure the influence intensity and interpretability of accumulated differences for each metabolite on the classification and discrimination of samples in each group. VIP $\geq 1$ is a common screening standard for differential metabolites. Figure 2 shows that samples in the two groups are on the left and right sides of the PLS-DA score map, respectively, indicating significant metabolic differences of samples between the two groups.

\section{Orthogonal partial least-squares discriminant analysis (OPLS-DA)}

OPLS-DA is a derivative algorithm of PLS-DA. Compared with PLS-DA, OPLS-DA integrates orthogonal signal correction (OSC) and PLS-DA, decomposing $\mathrm{X}$ matrix information into two types of information that are related and unrelated to Y. By removing unrelated differences, the related information is concentrated in the first predictive component. Subsequent model tests and screening of differential metabolites were analyzed with the use of OPLS-DA results. Figure 3 shows that the samples in the two groups are on the positive and negative sides of the first principal component, indicating that samples in the two groups have significant metabolic differences on the OPLS-DA score map.

\section{Plasma differential metabolites}

When combining multivariable statistical analyses (OPLS-DA VIP values) and univariable statistical analyses ( $t$ test $P$ values), significant differential metabolites between the two groups were screened. The threshold for significant differences was VIP $\geq 1$ and $P<0.05$.

There were six differential metabolites between the PSD and stroke groups, of which three metabolites were increased and three were decreased. Compared with the control group, p-chlorophenylalanine, phenylacetyl glutamine, and DHA levels were higher in the PSD group, while betaine (trimethylglycine), palmitic acid, and MHPG-SO 4 were decreased (Table 3).

\section{KEGG analysis}

Table 4 presents the pathways involving the differential metabolites identified above between the 10 stroke patients without PSD and 10 stroke patients with PSD. Palmitic acid is involved in the fatty acid elongation, fatty acid degradation, fatty acid metabolism, fatty acid biosynthesis, and biosynthesis of unsaturated fatty acids pathways. Betaine is involved in the glycine, serine, and threonine pathway. $\alpha-\mathrm{N}$-Phenylacetyl-L-glutamine is involved in the phenylalanine metabolism pathway. 


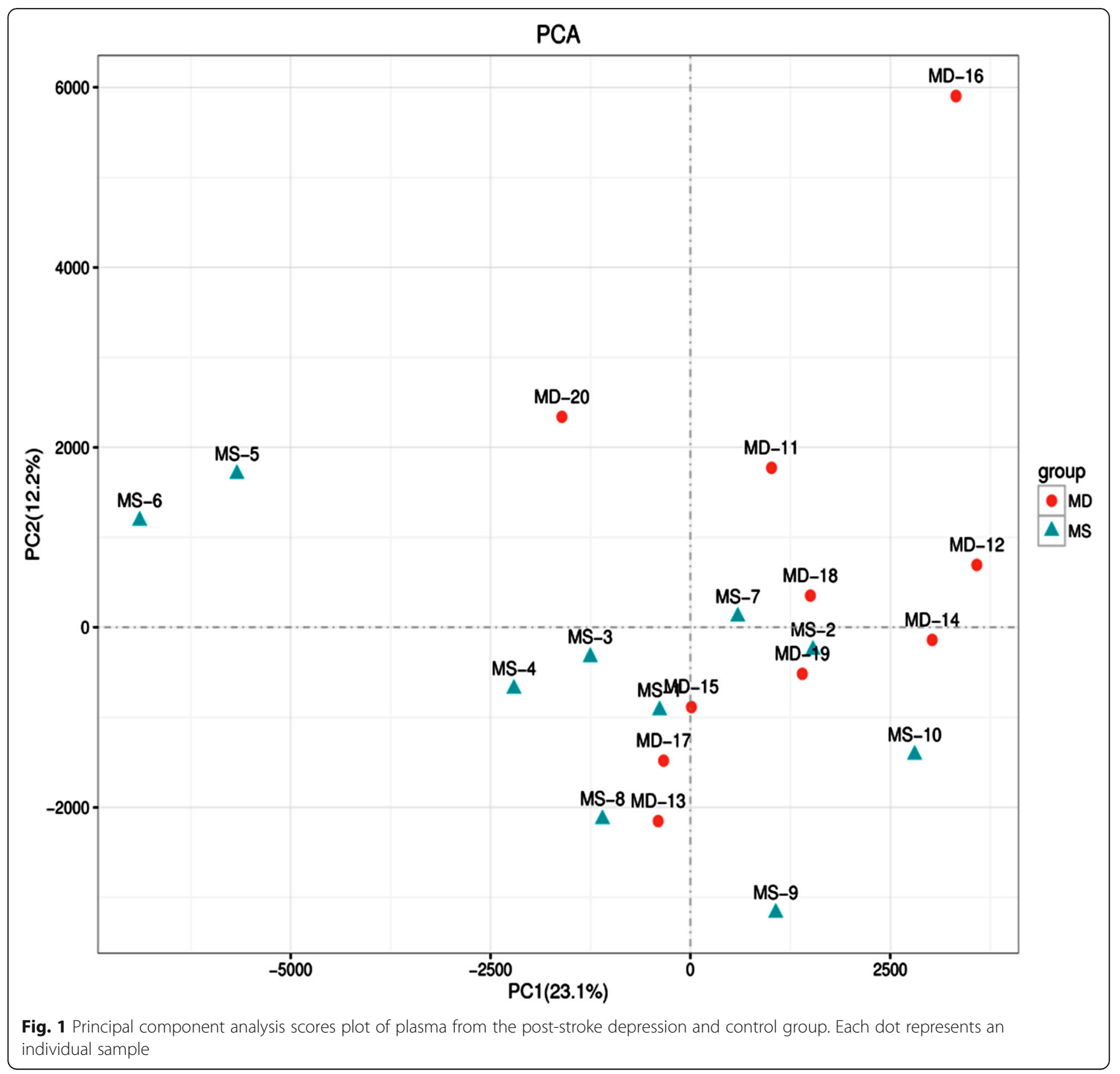

\section{Discussion}

In the present study, LC-MS-based metabolomics was used to analyze plasma metabolite profiles in PSD patients, and the metabolites related to PSD were screened. The KEGG was conducted for further analysis of metabolic pathways and the results suggest that plasma differential metabolites were predominantly related to amino acid metabolism (phenylalanine-tyrosine, tryptophan, methionine, and Hcy metabolisms), lipid metabolism, and oxidative stress.

Different methodologies will yield different results simply because they do not look at the same metabolites and those previous studies above are all based on GS/ MS. Indeed, Xiao et al. identified five potential biomarkers of PSD: lactic acid, $\alpha$-hydroxybutyric acid, phenylalanine, formic acid, and arabitol [14]. Zhang et al. found that azelaic acid, glyceric acid, pseudouridine, 5-hydroxycaproic acid, tyrosine, and phenylalanine can be used as biomarkers for the diagnosis of PSD [15]. Ding et al. identified proline, pyroglutamic acid, palmitic acid, oleic acid, linoleic acid, oxalic acid and rhamnose as being associated with PSD [16]. The differences between those three previous studies and the present study might be due to the sample preparation process and detection methods.

The differential metabolites associated with amino acid metabolism included phenylacetyl glutamine, pchlorophenylalanine, and trimethylglycine (betaine). 


\section{Scores (PLS-DA)}

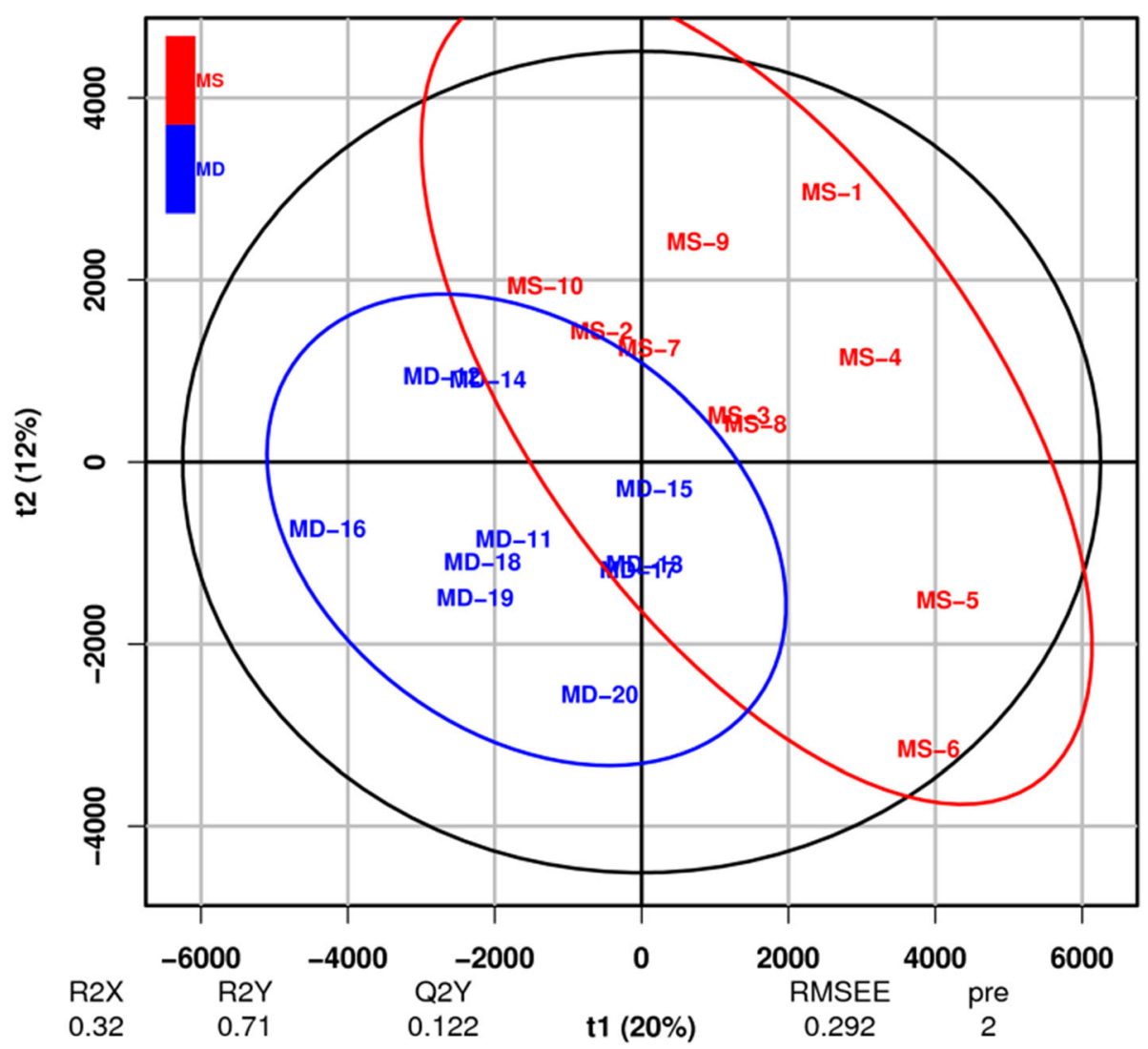

Fig. 2 PLS-DA scores plot of plasma from the post-stroke depression and control groups. Each dot represents an individual sample

Compared with the stroke group, levels of phenylacetyl glutamine and p-chlorophenylalanine were increased, while the levels of trimethylglycine were decreased in the PSD group.

Phenylacetyl glutamine is an important metabolite of phenylalanine. There are two main metabolic pathways for phenylalanine in human body: 1) phenylalanine transaminase catalyzes phenylalanine to phenylpyruvic acid, ultimately generating phenylacetyl glutamine; and 2) phenylalanine hydroxylase catalyzes phenylalanine to tyrosine. Tyrosine can be used to synthesize neurotransmitters such as dopamine and norepinephrine, which are directly related to the onset of depression. The up-regulation of phenylacetyl glutamine in the PSD group may be associated with imbalance of the two metabolic pathways of phenylalanine in the PSD group, with more phenylalanine being catalyzed into phenylacetyl glutamine rather than tyrosine, leading to depressive symptoms. Based on GCMS urine metabolomics, Zhang et al. found that levels of tyrosine and phenylalanine were decreased in the PSD group [15], supporting the present study and consistent with metabolic disorder of phenylalanine in the PSD.
Tryptophan is converted to serotonin via oxidative decarboxylation by tryptophan hydroxylase [21]. Tryptophan hydroxylase can be selectively inhibited by pchlorophenylalanine, resulting in a reduction in synthesis of serotonin [22]. Therefore, the up-regulation of $\mathrm{p}$ chlorophenylalanine levels in PSD in the present study may indirectly indicate a decrease in serotonin level in PSD.

Betaine is a highly active methyl donor and an alkaloid of quaternary ammonium salt, which is involved in the methionine-Hcy circulation. Betaine transfers methyl to Hcy through the betaine-Hcy methyltransferase (BHMT) in the liver, transformed into methionine and dimethylglycine, replacing methionine to provide methyl groups for other metabolic processes [23]. Methionine can react with ATP to form S-adenosylmethionine (SAM), which is the only methyl donor in the central nervous system, and it can promote the metabolism of neurotransmitters such as dopamine, serotonin, and norepinephrine [24]. Therefore, when betaine levels are decreased, due to the decrease of methyl donor, an increase in Hcy level, and a reduction in the production of methionine, the 


\section{Scores (OPLS-DA)}

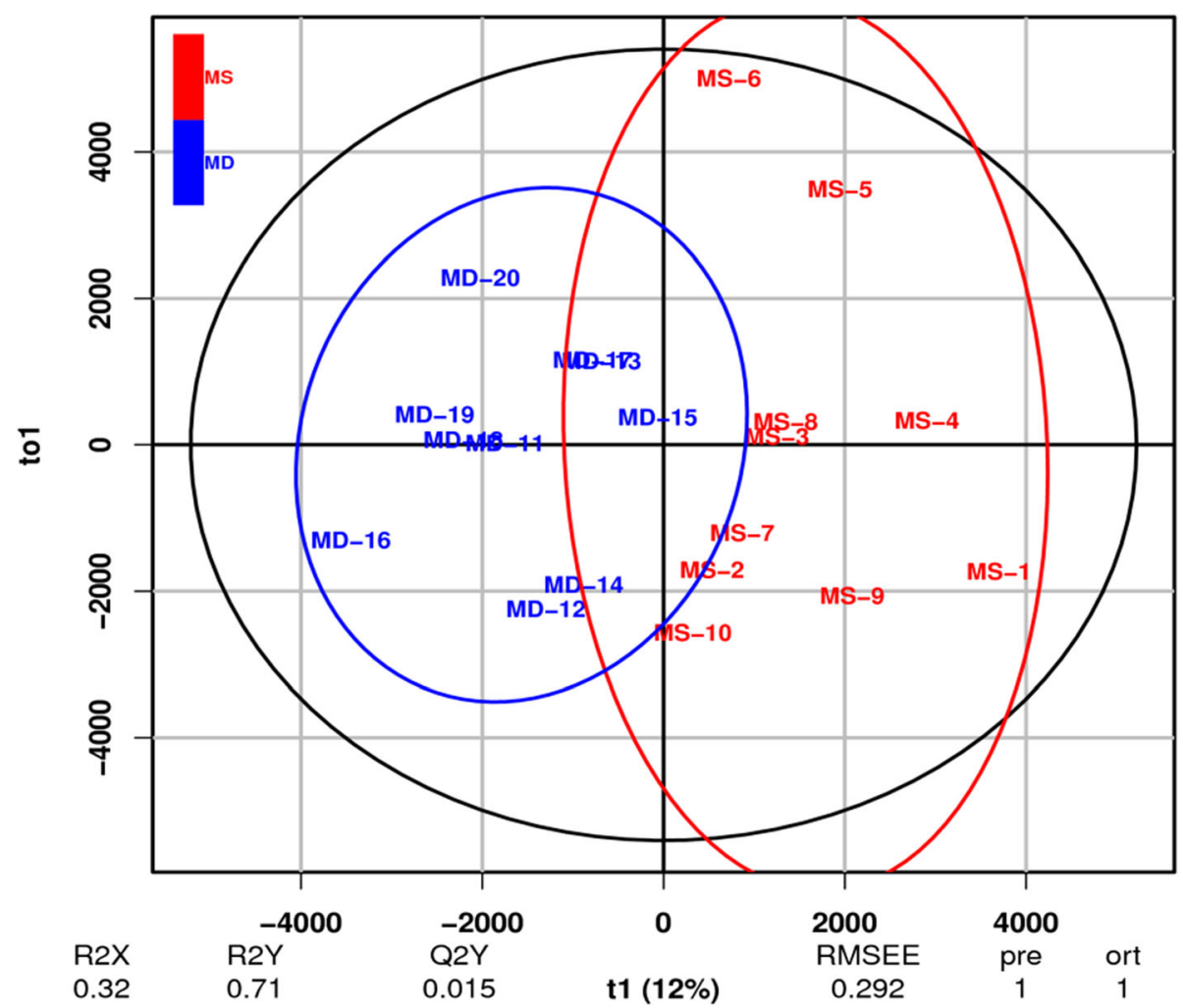

Fig. 3 OPLS-DA scores plot of plasma from the post-stroke depression and control groups. Each dot represents an individual sample

generation of dopamine, serotonin, and norepinephrine is reduced, resulting in depression. In addition, Hcy interferes with brain functions due to direct toxic effects on cerebral vascular endothelial cells, and it has toxic effects on depression-related nerve cells through various pathways, leading to depressive symptoms [24-26].

Among the differential metabolites screened, the KEGG analysis identified that the fatty acid elongation, fatty acid degradation, fatty acid metabolism, fatty acid biosynthesis, biosynthesis of unsaturated fatty acids pathways, the glycine, serine, and threonine pathway, and the phenylalanine metabolism pathways were probably involved in some manner in PSD. Nevertheless, those pathways are very complex and involve a number of metabolites, often intracellular, that were not detected in the present study. Additional studies are necessary to determine how those pathways are involved in PSD.

DHA is a long-chain $\omega-3$ polyunsaturated fatty acid and is associated with depression in many aspects. First, at the level of cell signal transduction, DHA is the predominant component of long-chain unsaturated fatty acids in cell membrane phospholipids, maintaining stability and fluidity of cell membrane, which plays an important role in cell signal transduction [27]. DHA has an

Table 3 Differential metabolites between the post-stroke depression and control groups

\begin{tabular}{lllllll}
\hline$\#$ & Compounds & RT & M/Z & VIP (OPLS-DA) & $P$ (t-test) & $\log _{2}$ FC \\
\hline 1 & a-N-Phenylacetyl-L-glutamine & 202.24 & 265 & 2.11 & 0.028 & 1.37 \\
2 & IS & 233.88 & 200 & 2.63 & 0.048 & 0.21 \\
3 & DHA & 36.67 & 327.23 & 1.47 & 0.011 & 0.77 \\
4 & Betaine & 286.68 & 118.08 & 1.43 & 0.043 & -0.79 \\
5 & Palmitic acid & 73.98 & 274.27 & 6.35 & 0.001 & -0.51 \\
6 & MHPG-SO $_{4}$ & 32.31 & 282.28 & 2.98 & 0.045 & -2.37 \\
\hline
\end{tabular}

$\log _{2} \mathrm{FC}$ is the logarithm of the mean ratio between the stroke group and post-stroke depression group. Positive numbers represent increase in the post-stroke depression group compared with the control group, and negative numbers mean a decrease 
Table 4 KEGG pathway analysis

\begin{tabular}{|c|c|c|c|c|c|}
\hline$\#$ & Pathway & $\begin{array}{l}\text { Pathway } \\
\text { ID }\end{array}$ & C_id & $\begin{array}{l}\text { Differentially expressed } \\
\text { metabolites }\end{array}$ & Description \\
\hline 1 & Fatty acid elongation & ko00062 & C00249 & 1021 & $\begin{array}{l}\text { Hexadecanoic acid; Hexadecanote; Hexadecylic acid; Palmitic acid; } \\
\text { Palmitate; Cetylic acid }\end{array}$ \\
\hline 2 & Fatty acid degradation & ko00071 & C00249 & 1021 & $\begin{array}{l}\text { Hexadecanoic acid; Hexadecanote; Hexadecylic acid; Palmitic acid; } \\
\text { Palmitate; Cetylic acid }\end{array}$ \\
\hline 3 & Fatty acid metabolism & ko01212 & C00249 & 1021 & $\begin{array}{l}\text { Hexadecanoic acid; Hexadecanote; Hexadecylic acid; Palmitic acid; } \\
\text { Palmitate; Cetylic acid }\end{array}$ \\
\hline 4 & $\begin{array}{l}\text { Glycine, serine and } \\
\text { threonine metabolism }\end{array}$ & ko00260 & C00719 & 90 & $\begin{array}{l}\text { Betaine; Trimethylaminoacetate; Glycine betaine; N,N,N- } \\
\text { Trimethylglycine; Trimethylammonioacetate }\end{array}$ \\
\hline 5 & Phenylalanine metabolism & ko00360 & $\mathrm{C} 04148$ & 945 & $\begin{array}{l}\text { Phenylacetylglutamine; alpha-N-Phenylacetyl-L-glutamine; N2-(2-Phe- } \\
\text { nylacetyl)-L-glutamine }\end{array}$ \\
\hline 6 & Fatty acid biosynthesis & ko00061 & C00249 & 1021 & $\begin{array}{l}\text { Hexadecanoic acid; Hexadecanote; Hexadecylic acid; Palmitic acid; } \\
\text { Palmitate; Cetylic acid }\end{array}$ \\
\hline 7 & $\begin{array}{l}\text { Biosynthesis of unsaturated } \\
\text { fatty acids }\end{array}$ & ko01040 & C00249 & 1021 & $\begin{array}{l}\text { Hexadecanoic acid; Hexadecanote; Hexadecylic acid; Palmitic acid; } \\
\text { Palmitate; Cetylic acid }\end{array}$ \\
\hline
\end{tabular}

effect on central neurotransmitters, especially the neural transmission or functions of serotonin and dopamine. Studies have shown that dopamine release in the brain regions of rats without long-chain $\omega-3$ polyunsaturated fatty acids is significantly lower than in controls [28]. Epidemiological data have demonstrated that small intake of fish products in depression patients is directly related to the depression, and that intake of long-chain $\omega-3$ polyunsaturated fatty acids is associated with a lower risk of suicide [29].

Second, DHA has an important effect on PPAR signaling pathways in inflammation. DHA can increase the expression levels and protein activity of PPAR- $\gamma$, and PPAR- $\gamma$ can inhibit NF- $\mathrm{KB}$ pathway after its activation, reducing the inflammatory response and promoting cell metabolism and mitochondrial function $[30,31]$. Studies have shown that mitochondrial morphological and functional abnormalities caused by oxidative stress may be related to depression, and mitochondrial dysfunction resulting from oxidative stress can change intracellular metabolism; lipid peroxidation is a notable metabolic characteristic in mitochondrial metabolic disorders, which is related to the psychopathology of MDD [32-34]. Oxidative stress occurs after stroke, and brain cells will consume a large amount of oxygen, producing massive amounts of free radicals. Given that brain tissue contains less antioxidants, high-density membrane unsaturated fatty acids, and higher oxidative metabolism rate, it is prone to be damaged by reactive oxygen species [35]. DHA can increase the levels of antioxidant enzymes, and by modifying the activity of superoxide dismutase (SOD), it can eliminate free radical produced after stroke and has a protective effect on the brain [36]. In the present study, the levels of DHA were higher in the PSD group compared with the control group. It is speculated that DHA in the PSD group is more effective in anti-oxidative stress injury after stroke than in promoting the release of neurotransmitters such as serotonin and dopamine. Ding et al. found that linoleic acid (a long-chain $\omega-6$ polyunsaturated fatty acid) and oleic acid (a monounsaturated $\omega-9$ fatty acid) were reduced in PSD based on GC-MS analysis [16]. Nevertheless, the association between metabolism disorders of unsaturated fatty acid and post-stroke depression needs further exploration.

Palmitic acid is the free saturated fatty acid with the highest level in plasma. Excessive consumption of fatty acids is often encountered in stroke patients, which is a reaction of restoring bio-energy balance state initiated by the protective regulatory system of the central nervous system when a decline in energy occurs due to stroke [37]. On the other hand, studies have shown that palmitic acid can activate $\mathrm{NF}-\mathrm{kB}$, promote massive release of inflammatory cytokines, and induce inflammatory responses of macrophages [38]. NF- $\mathrm{kB}$ is a key factor in connecting oxidative stress and inflammation [39]. In the present study, it was found that the plasma levels of palmitic acid were decreased in the PSD group, which was consistent with previous studies based on GC-MS methods [39]. Further research is needed to explore whether lower levels of palmitic acid is associated with related pathways of inflammation and oxidative stress, causing depressive symptoms, or lower levels are related to massive consumption and energy supply of fatty acids in PSD.

In amino acid metabolism (described above), betaine, as a methyl donor, is involved in the metabolism of methionine and Hcy, which may play a role in PSD. In addition, betaine, as a methyl donor, can also enhance carnitine synthesis and promote $\beta$-oxidation of long- 
chain fatty acids [40]. Some studies also showed that betaine can increase the activity of GSH-PX and SOD, eliminate oxygen free radicals, and play a protective role against oxidative stress damage of hippocampal neurons [13]. Multiple aspects could explain the decrease in betaine levels in the post-stroke depression group in this study and additional research is necessary to explain this.

The last differential metabolite was MHPG-SO , which showed a decrease in plasma levels in the PSD group compared with the control group. The main metabolite of norepinephrine in the brain is MHPG and it is combined with a sulfate group for urine excretion. In this study, the decrease in MHPG-SO ${ }_{4}$ levels in the PSD group could possibly be explained by a decrease in the concentration of adrenaline at synaptic cleft and a reduction in metabolism in PSD patients, further supporting the monoamine neurotransmitter hypothesis in PSD patients. Xiao et al. showed that compared with controls, the levels of plasma MPHG were significantly reduced in patients with depression before treatment, and MHPG levels showed an increase after effective antidepressant treatment [41].

The present study has limitations. Only 10 patients were included in each group, and the sample size was small. Nevertheless, due to stricter HDRS score for enrolled PSD patients, the smaller sample size was appropriate for metabonomics analyses. Therefore, larger sample size and multicenter studies are still needed to determine the biomarkers of PSD.

\section{Conclusions}

In the present study, metabolomics based on LC-MS was used to analyze the plasma metabolites in PSD patients. Six differential metabolites were screened, including phenylacetyl glutamine, p-chlorophenylalanine, betaine, DHA, palmitic acid and MHPG-SO ${ }_{4}$, suggesting that there are amino acid and lipid metabolism disorders in PSD patients, as well as increased oxidative stress in PSD.

\footnotetext{
Abbreviations

BDNF: Brain-derived neurotrophic factor; BHMT: Betaine-Hcy

methyltransferase; CT: Computed tomography; GS/MS: Gas chromatography/ mass spectrometry; Hcy: Homocysteine; HDRS: Hamilton depression rating scale; IL: Interleukin; INF: Interferon; LC-MS: Liquid chromatography-mass spectrometry; MRI: Magnetic resonance imaging; OPLS-DA: Orthogonal partial least-squares discriminant analysis; OSC: Orthogonal signal correction; PCA: Principal component analysis; PLS-DA: Partial least squares-discriminant analysis; PSD: Post-stroke depression; QC: Quality control; SAM: Sadenosylmethionine; SOD: Superoxide dismutase; TIC: Total ion curren; TNF: Tumor necrosis factor; VIP: Variable important in projection
}

\section{Authors' contributions}

MW, XWG conceived and coordinated the study, designed, performed and analyzed the experiments, wrote the paper. LXW, ST, HSW carried out the data collection, data analysis, and revised the paper. LX, WW designed the study, carried out the data analysis, and revised the paper. All authors reviewed the results and approved the final version of the manuscript.

\section{Funding}

This study was supported by grants from National Natural Science Foundation of China (Grant no.31860219) and the National Science Foundation of Jiangxi Province (Grant no.20151BAB205018, Grant no.20192BAB205047).

\section{Availability of data and materials}

The datasets used and/or analyzed during the current study are available from the corresponding author on reasonable request.

\section{Ethics approval and consent to participate}

This study was approved by the ethics committee of Nanchang University. All patients provided written informed consent to participate in this study. The study was carried out according to the tenets of the Declaration of Helsinki.

\section{Consent for publication}

Not applicable.

\section{Competing interests}

All authors declare that they have no competing interests.

Received: 28 August 2019 Accepted: 12 May 2020

Published online: 20 June 2020

\section{References}

1. American Psychiatric Association. DSM-5. Diagnostic and Statistical Manual of Mental Disorders-V. Washington, D.C.: American Psychiatric Association; 2015.

2. Robinson RG, Jorge RE. Post-stroke depression: a review. Am J Psychiatry. 2016;173:221-31.

3. Loubinoux I, Kronenberg G, Endres M, Schumann-Bard P, Freret T, Filipkowski RK, et al. Post-stroke depression: mechanisms, translation and therapy. J Cell Mol Med. 2012;16:1961-9.

4. Miller AH. Norman cousins lecture. Mechanisms of cytokine-induced behavioral changes: psychoneuroimmunology at the translational interface. Brain Behav Immun. 2009;23:149-58.

5. Zhu CB, Blakely RD, Hewlett WA. The proinflammatory cytokines interleukin1 beta and tumor necrosis factor-alpha activate serotonin transporters. Neuropsychopharmacology. 2006;31:2121-31.

6. Wang X, Li YH, Li MH, Lu J, Zhao JG, Sun XJ, et al. Glutamate level detection by magnetic resonance spectroscopy in patients with post-stroke depression. Eur Arch Psychiatry Clin Neurosci. 2012;262:33-8.

7. Masi G, Brovedani P. The hippocampus, neurotrophic factors and depression: possible implications for the pharmacotherapy of depression. CNS Drugs. 2011;25:913-31.

8. Li J, Zhao YD, Zeng JW, Chen XY, Wang RD, Cheng SY. Serum brain-derived neurotrophic factor levels in post-stroke depression. J Affect Disord. 2014;168: 373-9.

9. Li Y, Cao LL, Liu L, Qi QD. Serum levels of homocysteine at admission are associated with post-stroke depression in acute ischemic stroke. Neurol Sci. 2017;38:811-7.

10. Sanchez-Villegas A, Martinez-Gonzalez MA. Diet, a new target to prevent depression? BMC Med. 2013;11:3.

11. Guo $Z Y$, Tan $X Y$, Zhang $X N$. Analysis of proton magnetic resonance spectroscopy imaging on hippocampus in patients with post-stroke depression. Shandong Med J. 2014;54:20-4.

12. Bais P, Moon SM, He K, Leitao R, Dreher K, Walk T, et al. PlantMetabolomics. org: a web portal for plant metabolomics experiments. Plant Physiol. 2010; 152:1807-16.

13. Yang J, Chen T, Sun L, Zhao Z, Qi X, Zhou K, et al. Potential metabolite markers of schizophrenia. Mol Psychiatry. 2013;18:67-78.

14. Xiao J, Zhang J, Sun D, Wang L, Yu L, Wu H, et al. Discriminating poststroke depression from stroke by nuclear magnetic resonance spectroscopy-based metabonomic analysis. Neuropsychiatr Dis Treat. 2016;12:1919-25. 
15. Zhang W, Zhang XA. A novel urinary metabolite signature for non-invasive post-stroke depression diagnosis. Cell Biochem Biophys. 2015;72:661-7.

16. Ding X, Liu R, Li W, Ni H, Liu Y, Wu D, et al. A metabonomic investigation on the biochemical perturbation in post-stroke patients with depressive disorder (PSD). Metab Brain Dis. 2016;31:279-87.

17. Bouatra S, Aziat F, Mandal R, Guo AC, Wilson MR, Knox C, et al. The human urine metabolome. PLoS One. 2013;8:e73076.

18. Zheng YP, Zhao JP, Phillips M, Liu JB, Cai MF, Sun SQ, et al. Validity and reliability of the Chinese Hamilton depression rating scale. Br J Psychiatry. 1988;152:660-4.

19. Zhou B, Xiao JF, Tuli L, Ressom HW. LC-MS-based metabolomics. Mol BioSyst. 2012;8:470-81.

20. Xiao JF, Zhou B, Ressom HW. Metabolite identification and quantitation in LC-MS/MS-based metabolomics. Trends Analyt Chem. 2012;32:1-14.

21. Schrocksnadel K, Wirleitner B, Winkler C, Fuchs D. Monitoring tryptophan metabolism in chronic immune activation. Clin Chim Acta. 2006;364:82-90.

22. Hritcu L, Clicinschi M, Nabeshima T. Brain serotonin depletion impairs short-term memory, but not long-term memory in rats. Physiol Behav. 2007;91:652-7.

23. Craig SA. Betaine in human nutrition. Am J Clin Nutr. 2004;80:539-49.

24. Bottiglieri T. Homocysteine and folate metabolism in depression. Prog Neuro-Psychopharmacol Biol Psychiatry. 2005;29:1103-12.

25. Gilbody S, Lightfoot T, Sheldon T. Is low folate a risk factor for depression? A meta-analysis and exploration of heterogeneity. J Epidemiol Community Health. 2007;61:631-7.

26. Bhatia P, Singh N. Homocysteine excess: delineating the possible mechanism of neurotoxicity and depression. Fundam Clin Pharmacol. 2015;29:522-8.

27. Ying Z, Feng C, Agrawal R, Zhuang Y, Gomez-Pinilla F. Dietary omega-3 deficiency from gestation increases spinal cord vulnerability to traumatic brain injury-induced damage. PLoS One. 2012;7:e52998.

28. Zimmer L, Vancassel S, Cantagrel S, Breton P, Delamanche S, Guilloteau D, et al. The dopamine mesocorticolimbic pathway is affected by deficiency in n-3 polyunsaturated fatty acids. Am J Clin Nutr. 2002;75:662-7.

29. Mazza M, Pomponi M, Janiri L, Bria P, Mazza S. Omega-3 fatty acids and antioxidants in neurological and psychiatric diseases: an overview. Prog Neuro-Psychopharmacol Biol Psychiatry. 2007:31:12-26.

30. Mandrekar-Colucci S, Sauerbeck A, Popovich PG, McTigue DM. PPAR agonists as therapeutics for CNS trauma and neurological diseases. ASN Neuro. 2013;5:e00129.

31. Li H, Ruan XZ, Powis SH, Fernando R, Mon WY, Wheeler DC, et al. EPA and DHA reduce LPS-induced inflammation responses in HK-2 cells: evidence for a PPAR-gamma-dependent mechanism. Kidney Int. 2005;67:867-74.

32. Tobe EH. Mitochondrial dysfunction, oxidative stress, and major depressive disorder. Neuropsychiatr Dis Treat. 2013;9:567-73.

33. Milhavet $\mathrm{O}$, McMahon HE, Rachidi W, Nishida N, Katamine S, Mange A, et al. Prion infection impairs the cellular response to oxidative stress. Proc Natl Acad Sci U S A. 2000;97:13937-42.

34. Wu S, Zhou F, Zhang Z, Xing D. Mitochondrial oxidative stress causes mitochondrial fragmentation via differential modulation of mitochondrial fission-fusion proteins. FEBS J. 2011;278:941-54.

35. Basso M, Ratan RR. Transglutaminase is a therapeutic target for oxidative stress, excitotoxicity and stroke: a new epigenetic kid on the CNS block. J Cereb Blood Flow Metab. 2013;33:809-18.

36. Young G, Conquer J. Omega-3 fatty acids and neuropsychiatric disorders. Reprod Nutr Dev. 2005;45:1-28.

37. Belgardt BF, Bruning JC. CNS leptin and insulin action in the control of energy homeostasis. Ann N Y Acad Sci. 2010;1212:97-113.

38. Wu D, Liu J, Pang X, Wang S, Zhao J, Zhang X, et al. Palmitic acid exerts pro-inflammatory effects on vascular smooth muscle cells by inducing the expression of C-reactive protein, inducible nitric oxide synthase and tumor necrosis factor-alpha. Int J Mol Med. 2014;34:1706-12.

39. Huang B, Yang XD, Lamb A, Chen LF. Posttranslational modifications of NFkappaB: another layer of regulation for NF-kappaB signaling pathway. Cell Signal. 2010;22:1282-90.

40. Peluso G, Barbarisi A, Savica V, Reda E, Nicolai R, Benatti P, et al. Carnitine: an osmolyte that plays a metabolic role. J Cell Biochem. 2000;80:1-10

41. Xiao H, Yao H, Hou G. Depression and neurotransmitter metabolites in plasma. Nerv Dis Ment Health. 2002;2:209-10.

\section{Publisher's Note}

Springer Nature remains neutral with regard to jurisdictional claims in published maps and institutional affiliations.

Ready to submit your research? Choose BMC and benefit from:

- fast, convenient online submission

- thorough peer review by experienced researchers in your field

- rapid publication on acceptance

- support for research data, including large and complex data types

- gold Open Access which fosters wider collaboration and increased citations

- maximum visibility for your research: over $100 \mathrm{M}$ website views per year

At $\mathrm{BMC}$, research is always in progress.

Learn more biomedcentral.com/submissions 